\title{
Integrated photonic metasystem for image classifications at telecommunication wavelength
}

\section{Zi Wang}

University of Delaware https://orcid.org/0000-0003-1080-5825

\section{Lorry Chang}

University of Delaware

\section{Feifan Wang}

Peking University

\section{Tiantian Li}

University of Delaware

Tingyi Gu ( $\square$ tingyigu@udel.edu )

UNIVERSITY OF DELAWARE https://orcid.org/0000-0003-0414-6333

\section{Article}

Keywords: miniaturized image classifiers, photonic metasystem, telecommunication wavelength

Posted Date: May 20th, 2021

DOl: https://doi.org/10.21203/rs.3.rs-481200/v1

License: (c) (1) This work is licensed under a Creative Commons Attribution 4.0 International License. Read Full License

Version of Record: A version of this preprint was published at Nature Communications on April 19th, 2022. See the published version at https://doi.org/10.1038/s41467-022-29856-7. 


\title{
Integrated photonic metasystem for image classifications at telecommunication wavelength
}

\author{
Zi Wang, Lorry Chang, Feifan Wang, Tiantian Li and Tingyi Gu* \\ Department of Electrical and Computer Engineering, University of Delaware, Newark, DE \\ 19711, USA \\ *Email: tingyigu@udel.edu
}

Miniaturized image classifiers are potential for revolutionizing their applications in optical communication, autonomous vehicles, and healthcare. With deep diffractive neuron networks trained subwavelength structures, we demonstrate image recognitions by a passive silicon photonic metasystem. The metasystem implements high-throughput vector-by-matrix multiplications, enabled by $10^{3}$ passive subwavelength phase shifters as weight elements in $1 \mathrm{~mm}^{2}$ footprint. The large weight matrix size incorporates the fabrication variation related uncertainties, and thus the pre-trained metasystem can perform machine learning tasks without post-tuning. A 15-pixel spatial pattern classifier reaches near $90 \%$ accuracy with femtosecond inputs. The metasystem's superior parallelism $\left(10^{15}\right.$ bit/s) dramatically expand data processing capability of photonic integrated circuits, towards next generation low latency and low power photonic accelerators compatible with complementary metal-oxide-semiconductor manufacturing.

\section{Introduction}

Given the unparalleled throughput, low power consumption and scalability, spatial analog optical signal processors are perceived as a hardware accelerator for remote sensing, machine vision and holography [1-3]. With subwavelength control of the complex transmission coefficient, metamaterials open a new paradigm for the spatial signal processing [4-10]. The metamaterials perform mathematical operations through controlling interference and diffraction of the impinging electromagnetic wave by subwavelength structures [3, 11-15]. Fourier transfer method designed passive metalens systems demonstrate real-time spatial differentiation and edge detection [16-21]. Inversely designed nanophotonic components 
dramatically expands the functionality of the optical components with deterministic functions [14,22-24]. The computing capability in those systems are limited by their physical configurations and may not be sufficient for handling uncertainties that machine learning tasks require. High accuracy image classification and logic computing by the free-space optical network are demonstrated in millimeter and microwave waves [25-28]. Implementation of such multi-layer systems in infrared and visible wavelength is hindered by those systems' extreme sensitivity to interlayer misalignments [14,29-30].

Integrated photonic platform provides precise and robust interlayer alignment with lithographically defined patterns. Here high accuracy image classifications at telecommunication wavelength are demonstrated in a silicon photonic metasurface system. The cascaded layers of phase-only transmissive arrays are trained by deep diffractive neuron networks [8]. Compared to the waveguide based integrated photonic processors [31-33], the metasystem architecture offers three orders of magnitude higher throughput of vector-bymatrix multiplication (Supplementary Note S1) [34]. The metamaterial manifested weight element density, combined with diffraction strengthen inter-layer connectivity, enables the passive system to accomplish machine learning tasks of spatial pattern classification (Fig. 1ab). Also, the large data processing capacity incorporates the random nanofabrication offsets during the pre-training by deep diffractive neuron networks, and thus the passive photonic system is fully functional without active layers for phase correction. The broadband passive integrated metasystem can grasp the key information with a femtosecond single-shot exposure, and thus save the time and energy consumption for subsequent electronic processors. Spectral engineering is achieved in a three-layer metasystem for wavelength demultiplexing. The results indicates metasystem's potential for compressive sensing based hyperspectral imaging and machine vision $[29,35]$.

\section{Results}

The meta-system is defined on a silicon on insulator (SOI) substrate with a single-step lithography. Each phase shifter in the hidden layer is represented by two subwavelength slots, and the geometric parameters of the slots are learnable parameters. Each pair of the slots represents a weight element and connecting to following layers through diffraction and interference of the in-plane waves (Fig. 1b). Both amplitude modulation the phase shift of the 
transmitted wave can be programmed by adjusting the width and length of the subwavelength slots (Fig. 1c-d) [36]. With fixed slot width of $100 \mathrm{~nm}$, the phase of the transmission coefficient can be continuously tuned from 0 to $2 \pi$ with the slot length, while the amplitude stays more than $95 \%$. The incident angle is kept within the range of $\pm 30^{\circ}$ to ensure the amplitude of the transmission is more than $50 \%$. The phase distortion with oblique incidence is negligible.

The diffractive metasystem is firstly designed in Python and then verified by finite-difference time-domain (FDTD) simulations and experiments. During the training process, the phase shifts in each metasurface layer are iteratively updated by following the gradience decent algorithm (Supplementary Note 2) [37]. In the forward propagation step, we calculate outputs of the metasystem with input data (the training dataset), and then calculate the difference between the outputs of the metasystem and the target outputs (the loss of the neural network). In the backpropagation step, we calculate the gradient of the phase for every neuron and then update the phase value to decrease the loss of the neural network. The random phase noise with uniform distribution on the interval $[0,0.5 \pi)$ is added to the phase shift of each neuron during the training process to improve the system's robustness against nanofabrication variations. The photon propagation from layer $l$ with $k$ neurons to the next layer with $n$ neurons resemble the vector-matrix multiplication:

$$
\left[m^{l+1}(1), \quad \ldots, \quad m^{l+1}(n)\right]=\left[m^{l}(1) * t^{l}(1), \quad \ldots, \quad m^{l}(k) * t^{l}(k)\right] \cdot W
$$

where $t^{l}(p)=a * \exp \left[j \phi^{l}(p)\right]$ represents the transmission coefficient of the $p$-th neuron in $l$-th layer. The amplitude $a$ is near 1 for the slot width of $100 \mathrm{~nm}$. The phase shift $\phi^{l}(p)$ is proportional to the slot length. $m^{l}(p)$ and $m^{l+1}(q)$ are the amplitude of input photons towards the $p$-th neuron in the $l$-th layer and the $q$-th neuron in the $(l+1)$-th layer, respectively. The inter-layer connectivity $W$ is a $k \times n$ transfer matrix derived by the Rayleigh-Sommerfeld diffraction equation, representing the wave propagation in the SOI slab waveguide (Fig. 1b). The $(p, q)$-th element of the $W$ is $[25,38]$ :

$$
w(p, q)=\frac{\Delta y}{r^{2}}\left(\frac{1}{2 \pi r}+\frac{1}{j \lambda}\right) \exp \left(\frac{j 2 \pi r}{\lambda}\right)
$$

where $r$ is the distance between the $p$-th neuron in layer $l$ and the $q$-th neuron in layer $l+1 . \lambda$ is the effective wavelength in the planar waveguide.

As an example, we implement an integrated two-layer metasystem for letter image 
classifications. Each metasurface layer contains 450 phase shifters. The inter-layer distances are $100 \mu \mathrm{m}$. During the training process, the phase variations are incorporated (uniform distribution on the interval $[0,0.5 \pi))$ in each metasurface element for improving the systems' robustness to random fabrication offsets.

The metasystems and grating couplers are defined on the SOI substrate with single-step lithography and etching process (Methods). The setup for characterizing the metasystem is illustrated in Fig. 2a. The input patterns were reshaped from a two-dimensional (2D) matrix to a vector, and then projected onto the 1D grating coupler array through a digital micromirror device (DMD). The input patterns were the binary letter images with 15 pixels (Fig. 2b). The outputs were collected by a single-mode fiber through a grating coupler and delivered to a broadband infrared (IR) photodiode. A digital IR camera monitors the alignment between the input images and the grating coupler. Fig. 2c shows the perspective view of the device under test. The input grating couplers are one-dimensional (1D) gradient metasurface and about 10 $\mu \mathrm{m}$ wide in $\mathrm{x}$ direction (Fig. 2d). The scanning electron microscope (SEM) images illustrate partial area of the metasurface structure (Fig. 2e).

The testing dataset is the binary letter images with amplitude flipping in random pixels (Fig. $\mathrm{S} 1 \mathrm{~b})$. The two-layer metasystem was pre-trained by 10,000 such datasets. Numerical testing by the other 1000 testing dataset predicted $98 \%$ accuracy in letter classifications. Fig. 3a shows an example of the in-plane light distribution, representing an optical diffractive network (Fig. 3a). Three waveguides are on the output plane with $30 \mu \mathrm{m}$ spacing, representing the three letters in Fig. 2c. With an input image of letter ' $\mathrm{X}$ ', the light intensity onto channel 1 (Ch1) on the output plane. The light intensity distribution on the output plane is plotted as grey lines in Fig. 3b. The light intensity the position of Ch1 is clearly distinguished from the background. Channels 1,2 and 3 are correspondent to the letter ' $\mathrm{X}$ ', ' $\mathrm{Y}$ ' and ' $\mathrm{Z}$ ' respectively. The experimentally measured data (squares with error bars) are consistent with FDTD simulation (Fig. 3b). The blue, red and yellow squares are the light intensity from the grating couplers connected to Ch1, 2 and 3, respectively. We compare the confusion matrix of numerical testing (Fig. 3c) and measurement (Fig. 3d). Under continuous wave (CW) single wavelength excitation, the classification accuracy is measured to be $92 \%$. The broadband operation is numerically confirmed by FDTD simulation, and experimentally verified scanning the input 
CW laser across telecommunication C and L band (Supplementary Note S3). The broadband operation is critical for ensuring high classification accuracy for single-shot ultrafast pulsed inputs. Under 90 femtosecond pulsed light (centered at $1556 \mathrm{~nm}$ and bandwidth near $20 \mathrm{~nm}$ ), the measured confusion matrix shows $89 \%$ classification accuracy in the metasystem (Fig. 3e). The metasurface enabled large weight matrix size and its broadband operation allow the system throughput of $10^{15} \mathrm{~b} / \mathrm{s}$.

The dispersion of the metasurface system can be tailored for wavelength demultiplexer. We implemented a three-layer metasystem which can effectively separate input signal centered at 1490, 1530 and $1570 \mathrm{~nm}$ (Fig. 4). The distances between input plane, metasurfaces and output planes are fixed at $100 \mu \mathrm{m}$. Fig. 4a illustrates the FDTD simulated light distribution in plane. Three channel waveguides are placed on the output plane, with $30 \mu \mathrm{m}$ apart in x direction. Under $\mathrm{CW}$ tunable laser excitation at $1570 \mathrm{~nm}$, light merges at the position of the channel 3 waveguide. The numerical predicted spectra on the three channels (dashed curves in Fig. 4b) are compared to experiments (solid curves in Fig. 4b). The blue, red and orange curves representing the outputs for output waveguide channel 1, 2 and 3 respectively. FDTD simulations illustrate the full spectra of the wavelength dependent light intensity distribution on the output plane (Fig. 4c). The total loss of the three-layer metasystem is $15 \mathrm{~dB}$. It is noted that the complicated diffraction and interference allows one-to-one correspondence between the spatial distributions of the light and the laser wavelength. The spectral engineering capability is complementary to the spatial pattern classification for expanding device applications, such as compressive sensing-based spectrometers and hyperspectral imaging.

\section{Discussion.}

Compared to 2D metasurface in free-space systems, the number of weight elements in 1D metasurface is significantly reduced. However, the reduced weight matrix size does not compromise the system accuracy, attributing to efficient usage of those weight elements. Based on the Toeplitz circulant matrix, the training algorithm of the 1D metasystem requires less memory and time during the training process (Supplementary note S2). The phase only weight elements and the eliminated out-of-plane light propagation enables lower linear loss in the integrated metasystem. We numerically explored the metasysm's scalability by a designing one for a Modified National Institute of Standards and Technology (MNIST) handwritten digit 
database with 784-pixel inputs. 3 Epochs bring a metasystem's accuracy to be 96\% (Fig. S2). The only difference to the letter classification metasystem in Fig. 3 is that the MNIST system has one additional layer of metasurface.

The efficient design algorithm also facilities systematic design studies of the MNIST classifiers (Supplementary Note S4). Given sufficient weight matrix size, the system's accuracy can be fundamentally limited by the number of the metasurface layers (Fig. S3a). A one-layer metasystem can only achieve $88 \%$ accuracy. It increases to $92 \%$ in a two-layer system with more than 1500 weight elements per layer reaches. The maximum accuracy saturates at $96 \%$ in metasystems with more than two layers, as long as the total weight element is beyond 8,000. Longer inter-layer distance allows stronger inter-layer connectivity, and thus increases the metasystem accuracy (Fig. S3b).

Designed by diffractive neuron networks, we experimentally demonstrate cascaded metasurface systems for complexed spatial analog signal processing in telecommunication wavelength. The miniaturized image recognizing system is fabricated on SOI substrate with lithography defined alignment. Compared to conventional integrated photonic circuits, the manifested throughput and computing capability in such system is attributed to dense phase shifters and strong inter-layer connections. By introducing phase variations during the training process, the integrated metasystem can be robust against input noise and nanofabrication offsets. As a spatial pattern classifier, $92 \%$ and $89 \%$ accuracy were achieved in a two-layer metasystem, under narrow band CW excitation and broadband ultrafast laser excitation, respectively. The broadband operation of the pattern classifier allows single-shot image classification with boosted parallelism for optical signal processing. The wavelength selectivity of such metasystem can be co-designed with the pattern classification function for hyperspectral imaging, machine vision and hardware accelerators in optical interconnects.

\section{Methods}

Device fabrications. The integrated metasystem was fabricated on an SOI substrate from Soitec, with a $250 \mathrm{~nm}$ device layer and a $3 \mu \mathrm{m}$ thermal dioxide layer. The designed patterns (e.g. metasurface, waveguides, grating couplers) were firstly defined in CSAR 6200.09 positive resist layer by using a Vistec EBPG5200 electron beam lithography system with 100kV acceleration voltage, followed by optimized resist development and single-step dry etch 
procedures. A 300-nm thick silicon dioxide protection layer was finally deposited on the device layer by plasma-enhanced chemical vapor deposition (PECVD). The loss of grating couplers and channel waveguides used in the devices were less than $6 \mathrm{~dB}$ and $1 \mathrm{~dB}$ respectively.

Optical measurements. Tunable lasers (ANDO AQ4321A and AQ4321D) generate coherent and linearly polarized light with $1 \mathrm{pm}$ spectral resolution. For the pulsed signal measurement, a femtosecond laser centered at $1550 \mathrm{~nm}$ with a duration less than $90 \mathrm{fs}$ and spectral bandwidth around $50 \mathrm{~nm}$ (Calmar laser CFL-10CFF) is used to replace the continuous wave light source. The infrared light travels through a polarization controller, a beam expander, DMD (Texas Instruments DLP650LNIR), a lens, a long working distance objective (a Mitutoyo Plan Apo $20 \times$ infinity-corrected objective), and incident onto the input grating couplers. A single-mode fiber probe collects optical outputs and sends them to an InGaAs photodiode and optical power meter (Newport 818-IG-L-FC/DB and 1830-R-GPIB). A $640 \times 512$-pixel format and $25 \mu \mathrm{m}$ pitch size digital IR camera (Goodrich SU640KTSX) monitors the input pattern alignment with the substrate.

Numerical simulations. The integrated optical diffractive network is constructed in PyTorch framework (Supplementary note S2) [39] and verified by the 2D FDTD method.

\section{Acknowledgments}

This work was supported by AFOSR Young Investigator Program (FA9550-18-1-0300) and an Early Career Faculty grant from NASA's Space Technology Research Grants Program (80NSSC17K0526). The devices are fabricated at the University of Delaware Nanofabrication Facility with assistance from Dr. Kevin Lister.

\section{Author contributions}

Z.W. and T. G. conceived the idea. Z.W. developed the design principle and performed numerical simulations. Z.W. and T. L. fabricated the samples. Z.W., L.C, and F.W. built the experimental set-up. Z.W. performed the measurements and analyzed the data. Z.W. and T.G. wrote the manuscript with inputs from all authors.

\section{Competing interests}

The authors declare no competing for interest. 


\section{References}

[1] Solli, D. R. \& Jalali, B. Analog optical computing. Nat. Photonics 9, 704-706 (2015).

[2] Abdollahramezani, S., Hemmatyar, O. \& Adibi, A. Meta-optics for spatial optical analog computing. Nanophotonics 9, 4075-4095 (2020).

[3] Malkiel, I. et al. Plasmonic nanostructure design and characterization via deep learning. Light Sci. Appl. 7, 60 (2018).

[4] Chen, W. T., Zhu, A. Y. \& Capasso, F. Flat optics with dispersion-engineered metasurfaces. Nat. Rev. Mater. 5, 604-620 (2020).

[5] Khorasaninejad, M. et al. Metalenses at visible wavelengths: Diffraction-limited focusing and subwavelength resolution imaging. Science 352, 1190-1194 (2016).

[6] Arbabi, A., Horie, Y., Bagheri, M. \& Faraon, A. Dielectric metasurfaces for complete control of phase and polarization with subwavelength spatial resolution and high transmission. Nat. Nanotechnol. 10, 937-943 (2015).

[7] Faraji-Dana, M. et al. Compact folded metasurface spectrometer. Nat. Commun. 9, 4196 (2018).

[8] Wu, Z., Zhou, M., Khoram, E., Liu, B. \& Yu, Z. Neuromorphic metasurface. Photon. Res. 8, 46 (2020).

[9] Wang, S. et al. A broadband achromatic metalens in the visible. Nat. Nanotechnol. 13, 227232 (2018).

[10] Li, Y. et al. On-chip zero-index metamaterials. Nat. Photonics 9, 738-742 (2015).

[11] Silva, A. et al. Performing Mathematical Operations with Metamaterials. Science 343, 160-163 (2014).

[12] Mohammadi Estakhri, N., Edwards, B. \& Engheta, N. Inverse-designed metastructures that solve equations. Science 363, 1333-1338 (2019).

[13] Hughes, T. W., Williamson, I. A. D., Minkov, M. \& Fan, S. Wave physics as an analog recurrent neural network. Sci. Adv. 5, eaay6946 (2019).

[14] Backer, A. S. Computational inverse design for cascaded systems of metasurface optics. Opt. Express 27, 30308 (2019).

[15] Liu, D., Tan, Y., Khoram, E. \& Yu, Z. Training Deep Neural Networks for the Inverse Design of Nanophotonic Structures. ACS Photon. 5, 1365-1369 (2018).

[16] Zhou, J. et al. Optical edge detection based on high-efficiency dielectric metasurface. Proc. Natl. Acad. Sci. USA 116, 11137-11140 (2019).

[17] Zhang, J., Ying, Q. \& Ruan, Z. Time response of plasmonic spatial differentiators. Opt. Lett. 44, 4511 (2019).

[18] Kwon, H., Sounas, D., Cordaro, A., Polman, A. \& Alù, A. Nonlocal Metasurfaces for Optical Signal Processing. Phys. Rev. Lett. 121, 173004 (2018).

[19] Karimi, P., Khavasi, A. \& Mousavi Khaleghi, S. S. Fundamental limit for gain and resolution in analog optical edge detection. Opt. Express 28, 898 (2020).

[20] Rajabalipanah, H., Abdolali, A., Iqbal, S., Zhang, L. \& Cui, T. J. How Do Space-Time Digital Metasurfaces Serve to Perform Analog Signal Processing? arXiv:2002.06773 [physics] (2020).

[21] Wan, L. et al. Optical analog computing of spatial differentiation and edge detection with dielectric metasurfaces. Opt. Lett. 45, 2070 (2020).

[22] Yang, K. Y. et al. Inverse-designed multi-dimensional silicon photonic transmitters. 
arXiv:2103.14139 [physics] (2021).

[23] Ma, W. et al. Deep learning for the design of photonic structures. Nat. Photonics 15, 7790 (2021).

[24] Molesky, S. et al. Inverse design in nanophotonics. Nat. Photonics 12, 659-670 (2018).

[25] Lin, X. et al. All-optical machine learning using diffractive deep neural networks. Science 361, 1004-1008 (2018).

[26] Li, L. et al. Machine-learning reprogrammable metasurface imager. Nat Commun 10, 1082 (2019).

[27] Qian, C. et al. Performing optical logic operations by a diffractive neural network. Light Sci Appl 9, 59 (2020).

[28] Wetzstein, G. et al. Inference in artificial intelligence with deep optics and photonics. Nature 588, 39-47 (2020).

[29] Li, J. et al. Spectrally encoded single-pixel machine vision using diffractive networks. Sci. Adv. 7, eabd7690 (2021).

[30] Wang, Z., Chang, L., Wang, F., Li, T. \& Gu, T. Machine Learning with Integrated Metasystem. in Conference on Lasers and Electro-Optics SF2O.8 (OSA, 2020). doi:10.1364/CLEO_SI.2020.SF2O.8.

[31] Bogaerts, W. et al. Programmable photonic circuits. Nature 586, 207-216 (2020).

[32] Shastri, B. J. et al. Photonics for artificial intelligence and neuromorphic computing. Nat. Photonics 15, 102-114 (2021).

[33] Ying, Z. et al. Electronic-photonic arithmetic logic unit for high-speed computing. Nat. Commun. 11, 2154 (2020).

[34] Kitayama, K. et al. Novel frontier of photonics for data processing-Photonic accelerator. APL Photonics 4, 090901 (2019).

[35] Wang, Z. et al. Single-shot on-chip spectral sensors based on photonic crystal slabs. Nat. Commun. 10, 1020 (2019).

[36] Wang, Z. et al. On-chip wavefront shaping with dielectric metasurface. Nat. Commun. 10, 3547 (2019).

[37] Kingma, D. P. \& Ba, J. Adam: A Method for Stochastic Optimization. arXiv:1412.6980 (2014).

[38] Goodman, J. W. Introduction to Fourier optics. (Roberts \& Co, 2005).

[39] Paszke, A. et al. PyTorch: An Imperative Style, High-Performance Deep Learning Library. arXiv:1912.01703 (2019). 


\section{Figures}
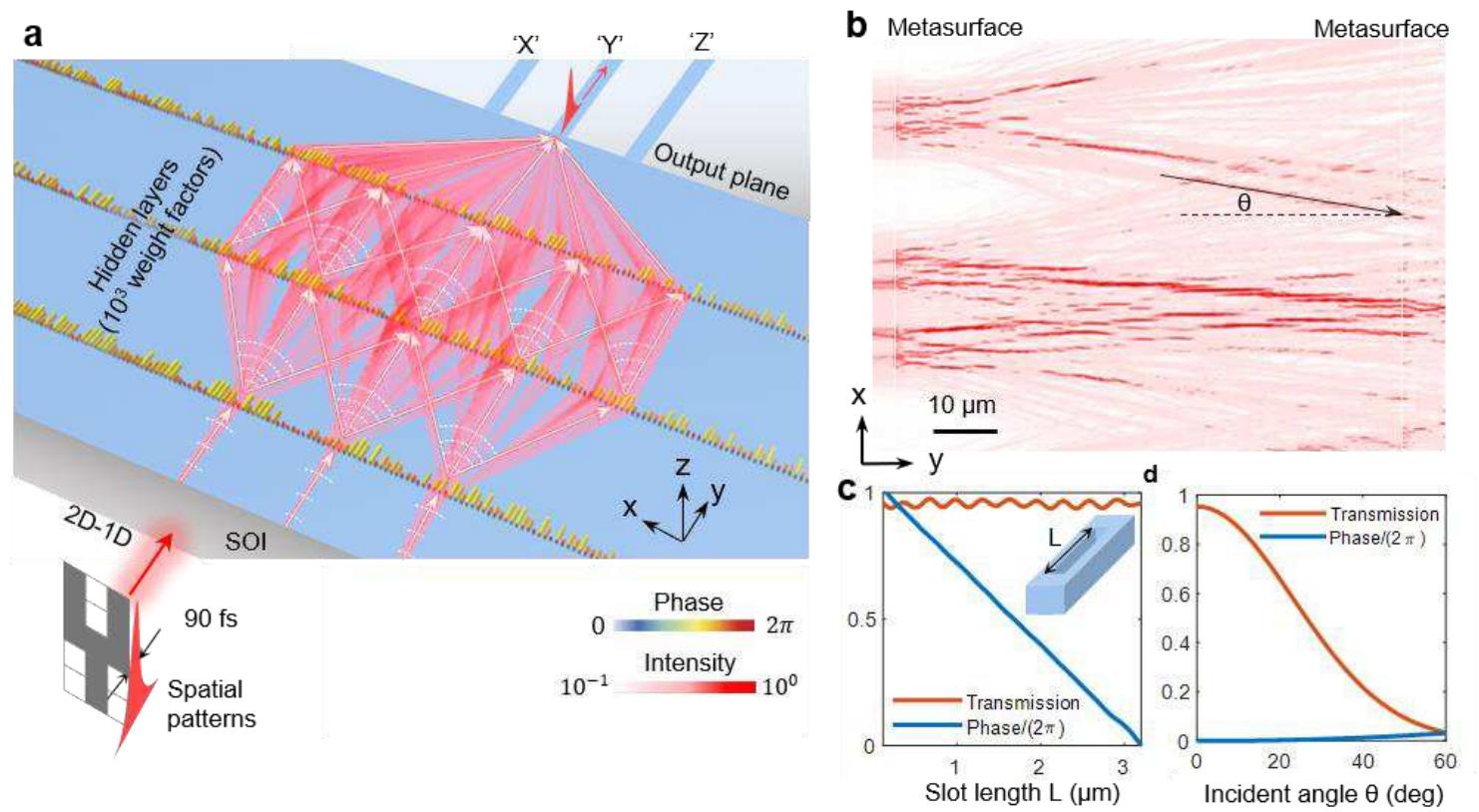

Figure 1. Integrated metasurface system for spatial pattern classification. (a) Schematics of the system architecture. As an example, an input pattern ' $\mathrm{Y}$ ' with ultrashort pulsed signal is coupled onto the chip. The diffractive optical network of metasurface systems process the signal. On the output plane, the light is converged onto the position where the correspondent waveguide channel locates. (b) Details of the light propagation in a diffractive optical network for strong inter-layer connectivity. (c) Amplitude and phase of the complex transmission coefficient versus the slot length (L) and (d) incident angle $(\theta)$ as indicated in b. 

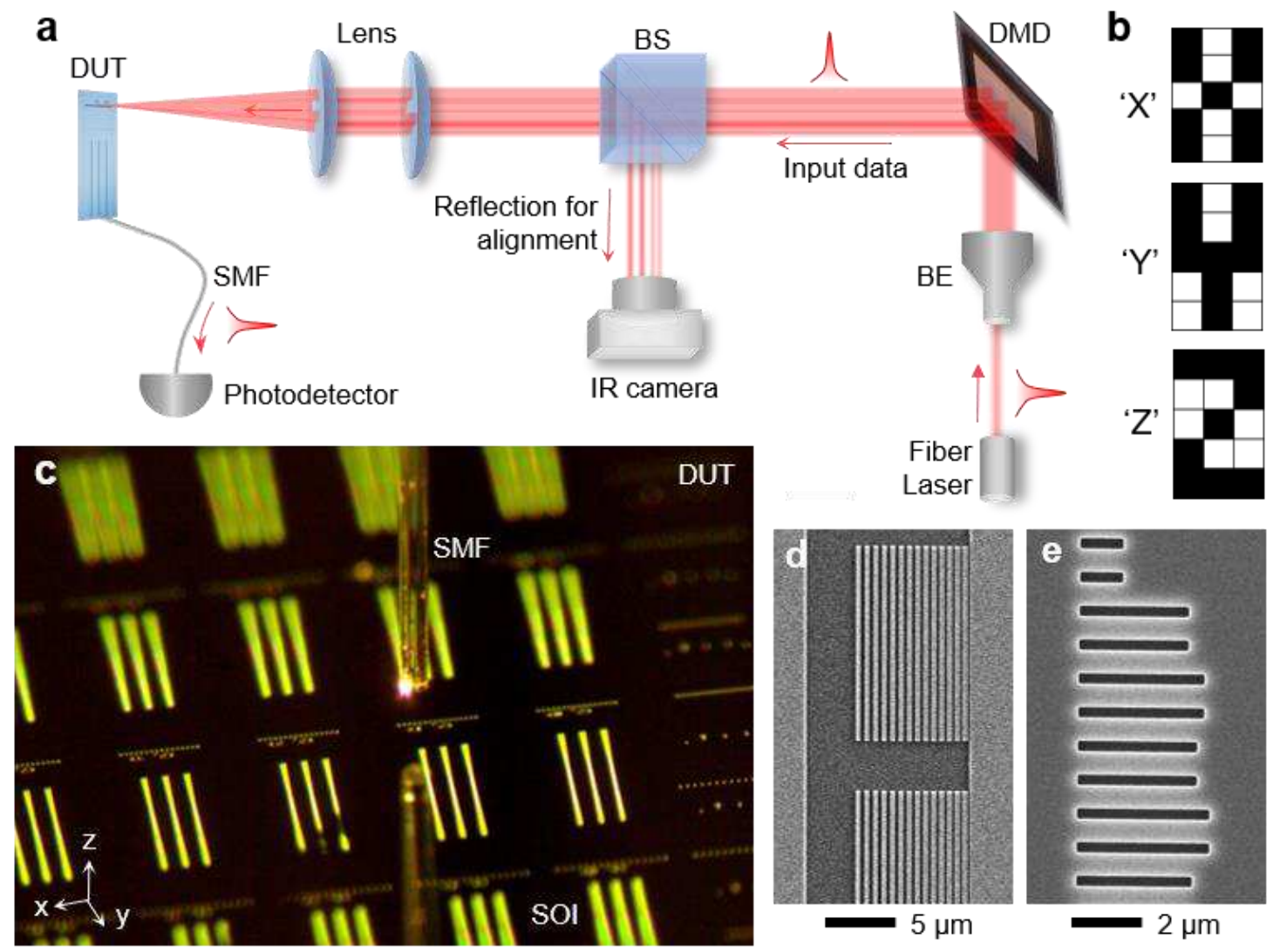

Figure 2. Device characterization. (a) The schematic illustration of the confocal set-up. The infrared laser beam is firstly expanded by the beam expander (BE). The reflection from the programmed micromirror device (DMD) carries the input pattern. The reflected image is then focused onto the input grating couplers arrays. The output signal is coupled to a single mode fiber (SMF) through a grating coupler. An IR camera is used to monitor the light coupling on and off the chip through a beam splitter (BS). (b) The input images for the letter ' $\mathrm{X}$ ', ' $\mathrm{Y}$ ' and ' $Z$ '. (c) The device under test (DUT) includes arrays of metasystems fabricated on a siliconon-insulator (SOI) substrate. (d) SEM images show the nanostructure of the input grating coupler array and (e) integrated metasurface. 

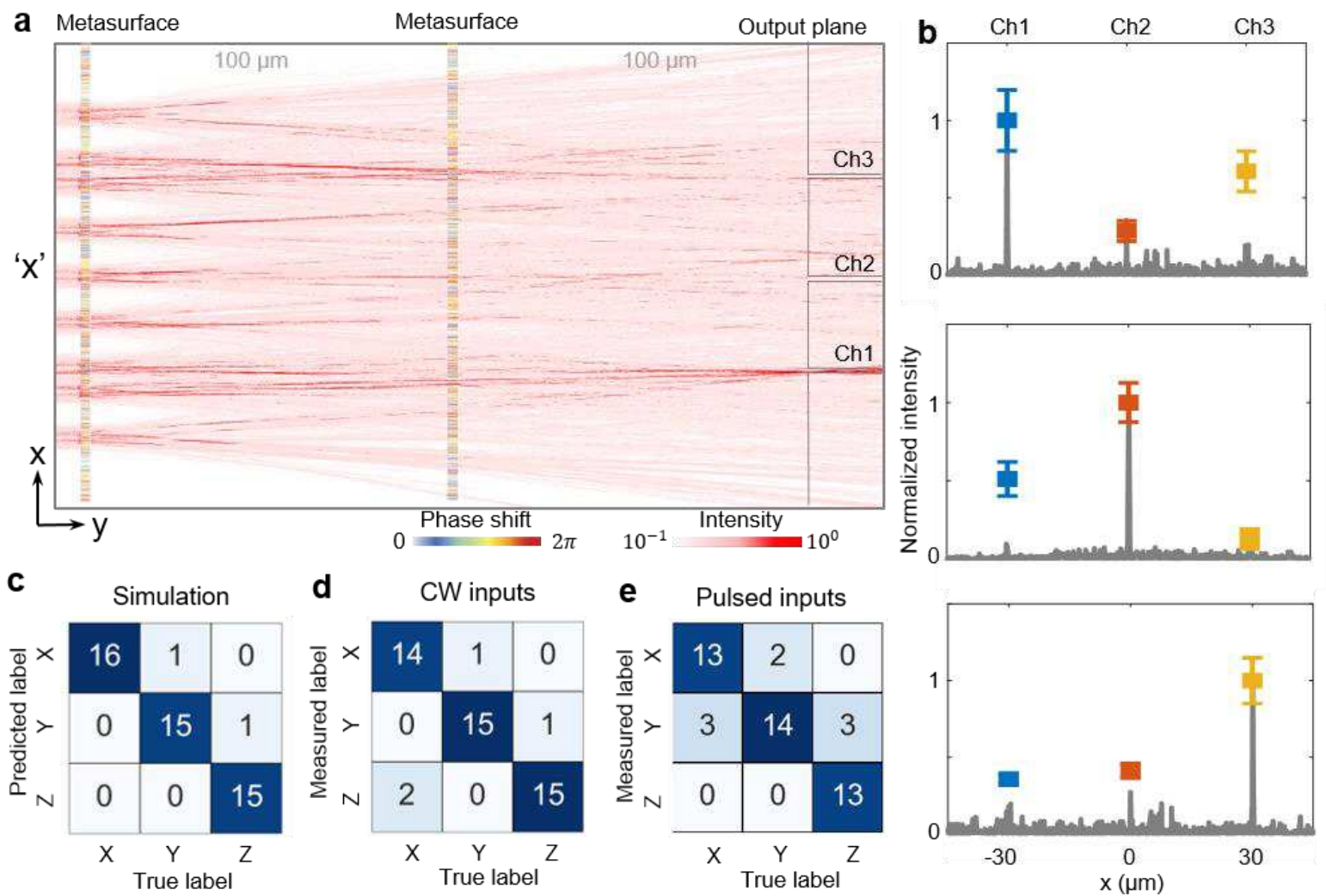

Figure 3. Broadband pattern classifier. (a) The in-plane electric field intensity distribution with the input patterns of "X" (log scale). (b) Comparison of measured optical intensities on the three waveguides placed on the output plane (dots with error bars) and the numerically simulated optical distribution on the output plane (grey curve). (c) Confusion matrix of FDTD simulated and (d) measured results for CW input with center wavelength of $1550 \mathrm{~nm}$ and bandwidth of $2 \mathrm{pm}$. (e) Measured confusion matrix for the femtosecond light source centered at $1556 \mathrm{~nm}$ and bandwidth over $30 \mathrm{~nm}$. 

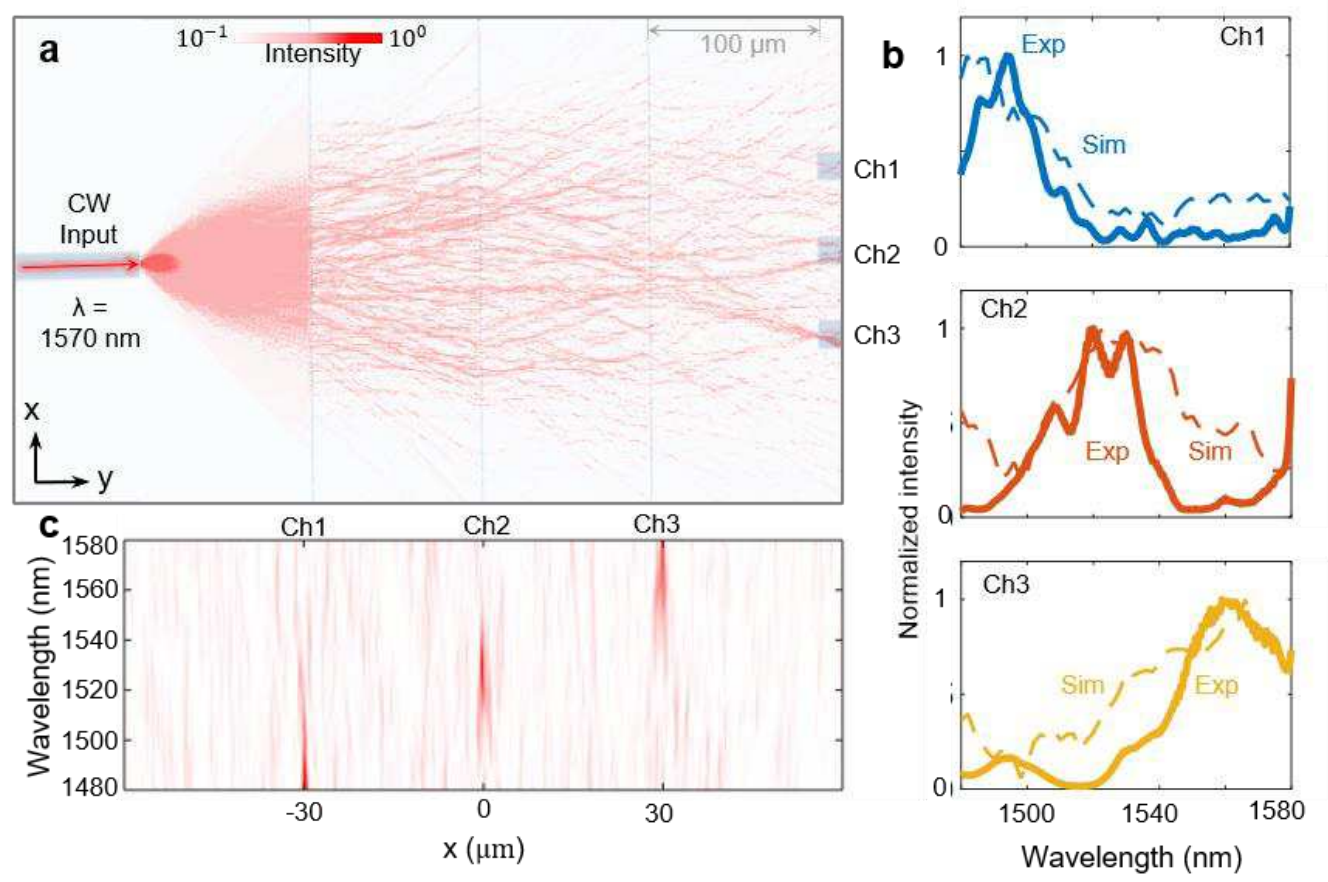

Figure 4. Dispersion engineered three-layer metasystem for wavelength demultiplication (a) The simulated in-plane light distribution superimposed on the optical image of a fabricated device. With input wavelength of $\lambda=1570 \mathrm{~nm}$, light paths merge near the position of channel 3 on the output plane. (b) Measured (solid lines) and simulated (dashed lines) spectra at three output ports. (c) Simulated optical intensity distributions on the output plane. Three waveguide output ports (Ch1, Ch2 and $\mathrm{Ch} 3)$ are centered at $\mathrm{x}=-30,0$ and $30 \mu \mathrm{m}$ respectively. 


\section{Supplementary Files}

This is a list of supplementary files associated with this preprint. Click to download.

- Supplement.docx 